\title{
capacité portante d'une semelle filante sur sol purement cohérent d'épaisseur limitée et de cohésion variable avec la profondeur
}

Massaad Matar

et 


\section{CAPACITE PORTANTE \\ D'UNE SEMELLE FILANTE SUR SOL $\Phi=0$ D'EPAISSEUR LIMITEE ET DE COHESION VARIABLE AVEC LA PROFONDEUR}

On étudie le problème de la capacité portante d'une semelle filante chargée axialement sur une couche de sol $\Phi=0$ d'épaisseur limitée, reposant sur une assise rigide, et dont la cohésion croît linéairement avec la profondeur.

Après avoir rappelé les résultats disponibles dans le cas soit de la couche de sol homogène reposant sur une assise rigide, soit du sol illimité dont la cohésion varie linéairement avec la profondeur. une première approche du problème est proposée s'appuyant sur la méthode de superposition et utilisant les résultats précédents.

Le calcul global de la capacité portante est ensuite effectué dans le cadre de la théorie des équilibres limites plans. Les résultats ainsi obtenus sont comparés à ceux fournis par la méthode de superposition, mettant en évidence l'effet majorateur du couplage entre cohésion en surface et gradient de cohésion.

Ces résultats sont facilement utilisables dans la pratique, par exemple pour le calcul de fondations sur sols marins, grâce à une présentation sous forme d'abaques.

\section{BEARING CAPACITY OF A STRIP FOOTING ON A $\Phi=0$ SOIL LAYER OF LIMITED THICKNESS WITH VARYING SHEAR STRENGTH}

The paper is concerned with the bearing capacity of a strip footing on a $\Phi=0$ soil layer of limited thickness resting on a rigid bed-rock, when the shear strength of the soil is supposed to increase linearly with depth.

The available results both for the case of a homogeneous soil layer of limited thickness resting on a rigid bed-rock, and for the case of an unlimited soil with linearly increasing shear strength, are first recalled. Thence, by means of the superposition method, a first approach of the problem under consideration is proposed, making use only of the afore-exposed results.

A global calculation of the bearing capacity is then performed, taking into account the effects of surface load, cohesion and cohesion gradient simultaneously, within the frame of the plane limit equilibrium theory. The so-obtained results being compared with those given by the superposition method, it is pointed out that the bearing capacity is underestimated through the use of the superposition method, due to the coupling effact between the surface cohesion and the cohesion gradient.

Plotting the results into adequate charts make their use easy for practical purposes, for instance when dealing with foundations on a marine subsoil. 


\title{
capacité portante d'une semelle filante sur sol purement cohérent d'épaisseur limitée et de cohésion variable avec la profondeur
}

\author{
par Massaad MATAR et Jean SALENÇON
}

\section{NOTATIONS}

a : largeur de la zone déformée plastiquement en surface

B : largeur de la fondation

C : cohésion constante (sol homogène)

$\mathrm{C}(\mathrm{z})$ : cohésion variable avec la profondeur

$\mathrm{C}^{\circ}$ : cohésion en surface

$\mathrm{F}$ : force portante de la fondation

$\mathrm{F}_{c}\left(g^{o} \mathrm{~B} / \mathrm{C}^{o}\right)$ : coefficient du terme de cohésion (sol non homogène, illimité)

$F_{c}\left(\mathrm{~B} / h, g^{\circ} \mathrm{B} / \mathrm{C}^{\circ}\right)$ : coefficient du terme de cohésion (couche de sol non homogène, épaisseur limitée)

$g^{\circ} \quad$ : gradient vertical descendant de cohésion $g^{o}=-d \mathrm{C} / d z$

$h \quad$ : épaisseur de la couche

$h_{o} \quad$ : épaisseur de la zone perturbée (sol non homogène, illimité)
$\mathrm{N}_{c}$ : coefficient du terme de cohésion (sol homogène, illimité) : $\mathrm{N}_{c}=\pi+2$

$\mathrm{N}_{c}^{\prime}(\mathrm{B} / h)$ : coefficient du terme de cohésion (couche du sol homogène, épaisseur limitée)

$G_{c}\left(\mathrm{~B} / h, g^{o} h / \mathrm{C}^{o}\right)$ : coefficient du terme de cohésion couche du sol non homogène, épaisseur limitée)

$p \quad:$ " pression moyenne » $p=-\left(\sigma_{1}+\sigma_{2}\right) / 2$

$p_{\text {ult }}$ : capacité portante de la fondation : $p_{\text {ult }}=\mathrm{F} / \mathrm{B}$

$\alpha, \beta$ : lignes caractéristiques

$\gamma \quad$ : poids spécifique du sol

$\Phi$ : angle de frottement interne du sol : $\Phi=0$

$\sigma_{1}, \sigma_{2}$ : contraintes principales (tractions positives) : $\sigma_{1} \geqslant \sigma_{2}$

$\theta \quad$ : angle de la traction maximale avec $O x$ : $\theta=\left(\overrightarrow{O x}, \overrightarrow{\sigma_{1}}\right)$

\section{INTRODUCTION}

La capacité portante des fondations superficielles sur sol non-homogène, a fait l'objet de nombreuses études théoriques et expérimentales. Deux types principaux de non-homogénéité ont été essentiellement abordés :

d'une part le cas de la fondation sur une couche de sol homogène d'épaisseur limitée, reposant sur une assise rigide ;

d'autre part, le cas de la fondation sur sol illimité dont la cohésion varie avec la profondeur; pour des raisons pratiques, et en particulier pour pouvoir présenter les résultats sous forme d'abaques, seul le cas de la cohésion croissant linéairement avec la profondeur a été traité.

L'importance pratique de tels problèmes de nonhomogénéité est évidente. Le premier type se rencontre aussi bien pour les sols purement cohérents $(\Phi=0)$ que pour les sols frottants $(\Phi \neq 0)$, le second se pose le plus souvent dans le cas des sols à $\Phi=0$ ou $\Phi$ voisin de zéro, tels que les sols marins.

L'étude présentée ici, traite globalement dans le cas $\Phi=0$, les deux types de non-homogénéité : on a affaire à une couche de sol d'épaisseur limitée reposant avec frottement maximal sur une assise rigide et dont la cohésion croît linéairement avec la pro- fondeur. En l'état actuel, lorsque ce type de problème se pose (ce peut être le cas par exemple lorsque l'on a affaire à une fondation de grande largeur sur sol maritime), il ne peut être traité que par des errements tels que :

- traiter la couche comme limitée et homogène en ne prenant en compte que la cohésion en surface ;

- traiter le sol comme illimité et non homogène ;

- traiter la couche comme limitée et homogène en prenant en compte une cohésion " moyenne ";

- traiter la couche limitée et non homogène en cumulant la majoration due à la présence de l'assise rigide dans le cas du sol homogène d'une part, et celle due au gradient de cohésion dans le cas d'un sol illimité, de l'autre.

Il est pratiquement évident par le bon sens (et ceci est confirmé par la théorie exposée dans la suite) que les deux premières façons de procéder vont dans le sens de la sécurité ; elles peuvent aussi (surtout pour la première d'entre elles) conduire à des dimensionnements inutilement abondants; les deux dernières méthodes par contre risquent d'être trop optimistes.

Les résultats obtenus par l'étude globale, outre l'intérêt primordial de pouvoir être appliqués directement, présenteront aussi celui de permettre de situer les méthodes évoquées ci-dessus. 


\section{FONDATION SUR UNE COUCHE DE SOL $\Phi=0$ HOMOGENE D'EPAISSEUR LIMITEE}

L'étude théorique de la capacité portante d'une fondation chargée axialement sur une couche de sol homogène d'épaisseur limitée reposant sur une assise rigide, fait l'objet d'une bibliographie importante. Comme pour tous les problèmes de capacité portante, les méthodes de calcul employées relèvent du calcul à la rupture. Dans le cas du sol purement cohérent $(\Phi=0)$ qui nous intéresse ici, l'interprétation des résultats obtenus ne présente pas de difficulté : le sol est en effet alors représenté par un matériau de Tresca pour lequel on sait que le principe du travail plastique maximal est acceptable ; le calcul à la rupture s'identifie à la théorie classique des charges limites (voir par exemple [24]).

Une solution statique, c'est-à-dire la mise en évidence dans tout le système (sol de fondation et interfaces de contact) d'un champ de contraintes statiquement admissible et respectant le critère de plasticité ( $\left.{ }^{1}\right)$, conduit à une minoration de la capacité portante ;

Une solution cinématique, c'est-à-dire la mise en évidence d'un champ de vitesses cinématiquement et plastiquement admissible dans tout le système, conduit à une évaluation par excès de la capacité portante.

D'autre part, s'agissant d'une fondation superficielle sur un sol d'angle de frottement interne nul, on démontre sans difficulté que le poids spécifique $\gamma$ du sol n'intervient pas dans l'expression de la capacité portante tandis que la surchage $q \mathrm{y}$ apparaît sous forme purement additive $\left(^{2}\right)$.

Button [3] a étudié le problème pour la semelle filante, au moyen de lignes de rupture circulaires; le résultat obtenu s'interprète donc comme une majoration de la capacité portante.

Meyerhof et Chaplin [14] ont, pour le même problème, construit une solution par la théorie des équilibres limites plans; le champ de vitesses correspondant a été présenté par Johnson et Kudo [11], et le champ de contraintes a été complété dans les zones rigides par Salençon [20], Cette solution, " complète " au sens de Bishop [2], fournit donc la valeur exacte de la capacité portante théorique d'une semelle filante sur couche de sol $\Phi=0$, d'épaisseur limitée reposant avec adhérence totale sur une assise indéformable; le contact entre la semelle et le sol de fondation y est supposé se faire avec frottement maximal, c'est-à-dire qu'un glissement mobilise toute la résistance à la cission du sol.

B désignant la largeur de la fondation et $h$ l'épaisseur de la couche (fig. 1), cette solution ne prend effet que pour $\mathrm{B} / h \geqslant \sqrt{2}$. Pour $\mathrm{B} / h \leqslant \sqrt{2}$, la solution complète du problème est constituée par la solution de Prandtl (1923), que l'on peut prolonger dans les zones non déformées jusqu'à l'interface $\left({ }^{1}\right)$, par la méthode de Shield [28] (voir fig. 6) : ainsi pour $\mathrm{B} / h \leqslant \sqrt{2}$ l'assise rigide n'a pas d'influence. Avec les notations usuelles $q$ désignant la surcharge uniforme de surface, $C$ la cohésion du sol et $p_{\text {ult }}=\mathrm{F} / \mathrm{B}$ la capacité portante, celle-ci a alors la forme classique :

$$
p_{\text {ult }}=q+(\pi+2) \mathrm{C} \text {. }
$$

Mandel et Salençon [15] ont examiné le cas extrême où le contact entre la couche de sol et l'assise rigide se ferait sans frottement, mettant en particulier en évidence une diminution de la capacité portante par rapport au cas du sol homogène, sur une certaine plage de valeurs de $\mathrm{B} / h$.

La capacité portante se met alors sous la forme :

$$
p_{\text {ult }}=q+\mathrm{CN}_{c}^{\prime}\left(\frac{\mathrm{B}}{h}\right)
$$

L'abaque des valeurs de $\mathrm{N}_{c}^{\prime}$ obtenues par la solution exacte dans le cas où le contact entre sol et assise rigide est à adhérence totale, et par les solutions de

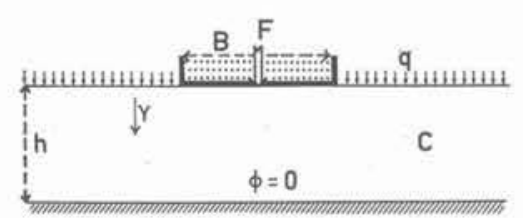

Fig. 1. - Semelle filante chargée axialement sur une couche de sol d'épaisseur limitée.
Fig. 2. - Valeurs de $\mathbf{N}^{\prime}$ dans le cas de l'interface "rugueux " et de l'interface $\propto$ lisse $»$.

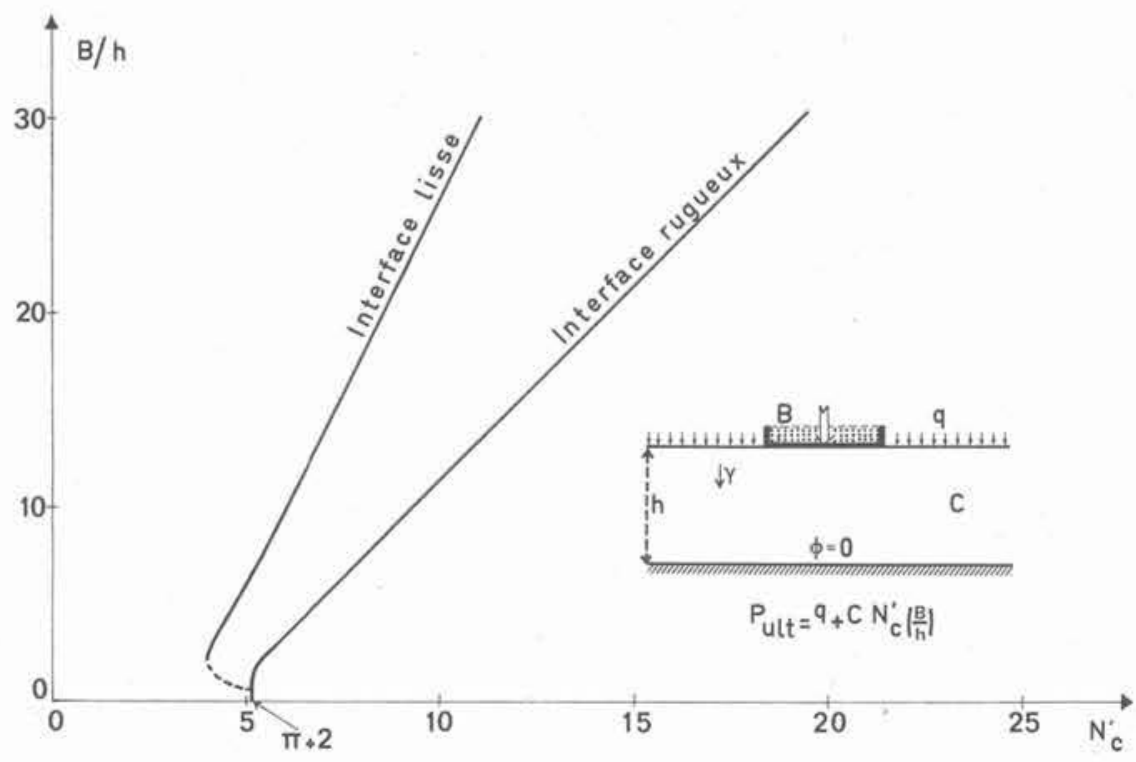

(') On vérifie que la contrainte normale sur l'interface est bien compressive : pas de décollement [20].
(') On dit aussi : plastiquement admissible.

$\left.{ }^{2}{ }^{2}\right)$ Ce résultat reste valable dans le cas du matériau non homogène. Pour cette raison on ne traitera dans toute la suite que le cas du sol non-pesant. 
Mandel et Salençon dans le cas où ce contact se ferait sans frottement, est donné à la figure 2 ; dans ce dernier cas la partie pointillée de la courbe indique que la valeur obtenue pour $\mathrm{N}_{c}^{\prime}$ n'est alors en toute rigueur qu'une majoration du facteur de cohésion (on se reportera à [15] pour une discussion détaillée).

Il semble que dans la majorité des cas pratiques, il y ait lieu de considérer que le contact entre sol de fondation et assise rigide s'effectue avec frottement maximal; c'est donc la seule hypothèse que nous retiendrons pour la suite. Signalons qu'alors on peut ( $\left.{ }^{1}\right)$, dans les utilisations courantes, adopter dès que $\mathrm{B} / h \geqslant 2$ la représentation asymptotique de $\mathrm{N}_{c}^{\prime}(\mathrm{B} / h)$ :

$$
\mathrm{N}_{c}^{\prime}(\mathrm{B} / h)=\pi+1+\frac{1}{2} \mathrm{~B} / h=4.1+0.5 \mathrm{~B} / h .
$$

Signalons enfin que pour la fondation axisymétrique sur une couche de sol $\Phi=0$ d'épaisseur limitée reposant sur une assise rigide, le calcul de la capacité portante a été effectué par Salençon, Croc, Michel et Pecker [21] en adoptant l'hypothèse de HaarKarman sur la forme du champ de contraintes; on a ainsi obtenu la valeur de $\mathrm{N}_{c}^{\prime}(\mathrm{B} / h)$ pour la fondation " rugueuse " avec interface " rugueux " mettant en évidence que la majoration de la capacité portante due à la présence de l'assise rigide par rapport au cas du sol illimité (Eason et Shield [6]) est moindre pour la fondation circulaire que pour la semelle filante.

\section{FONDATION SUR SOL $\Phi=0$ ILLIMITE DE COHESION VARIABLE AVEC LA PROFONDEUR}

\subsection{Solutions cinématiques}

Le problème de la capacité portante d'une semelle filante chargée axialement sur sol illimité $\Phi=0$, de cohésion croissant linéairement avec la profondeur, semble avoir été étudié pour la première fois par Kuznetzov [13] qui a proposé une solution approximativement cinématique dans le cas d'une fondation * lisse ". Le caractère de majorant du résultat obtenu a été confirmé par une solution cinématique différente donnée par Salençon ([19] et [20]). Avec les notations de la figure $3, g^{\circ}$ désignant le gradient vertical descendant de cohésion, $q$ la surcharge, $\mathrm{C}^{\circ}$ la cohésion en surface, la cohésion est de la forme :

$$
\mathrm{C}(z)=\mathrm{C}^{\circ}-g^{\circ} z
$$

et la majoration pour la capacité portante est donnée par la formule linéaire :

$$
p_{\text {uit }} \leqslant q+(\pi+2) \mathrm{C}^{o}+g^{o} \mathrm{~B}
$$

De même pour la fondation " rugueuse », Salençon ([19] et [20]) a obtenu les majorations :

$$
p_{\mathrm{ult}} \leqslant q+(\pi+2) \mathrm{C}^{o}+2 g^{o} \mathrm{~B} \quad \text { si } g^{o} \mathrm{~B} / \mathrm{C}^{o} \leqslant \frac{1}{2},
$$

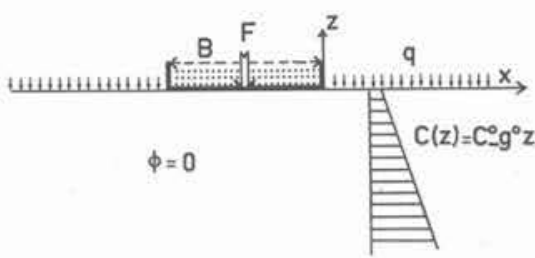

Fig, 3. - Fondation sur sol $\Phi=0$ de cohésion croissant linéairement avec la profondeur.

Fig. 4. - Valeurs de $\mathbf{F}_{\mathrm{c}}$ dans le cas de la semelle « rugueuse » et de la semelle "lisse ».
(3.4)

$$
\begin{aligned}
p_{\text {ult }} \leqslant q+(\pi+3) \mathrm{C}^{\circ}+g^{\circ} \mathrm{B}-\frac{1}{4}\left(\mathrm{C}^{o}\right)^{2} / g^{\circ} \mathrm{B} & \\
& \text { Si } g^{\circ} \mathrm{B} / \mathrm{C}^{o} \geqslant \frac{1}{2} .
\end{aligned}
$$

Des calculs cinématiques, utilisant des lignes de glissement circulaires, ont également été effectués, (Button [3] ; Giroud, Tran Vo Nhiem et Obin [10]) fournissant une majoration de la capacité portante de la fondation de la forme :

(3.5) $\quad p_{\text {ult }}=q+\mathrm{C}^{o} \mathrm{~N}_{c z}^{o}$

où $\mathrm{N}_{c z}^{o}$ est donné par une table et un abaque.

\subsection{Solution complète pour $C^{\circ} \neq 0$}

L'étude de ce type de problème en s'appuyant sur la théorie des équilibres limites plans a été faite par Favretti ([7], [8] et [9]) dans le cas où la cohésion décroît avec la profondeur. Berthet, Hayot et Salençon [1] ont construit la solution dans le cas de la cohésion croissante et non nulle en surface; cette

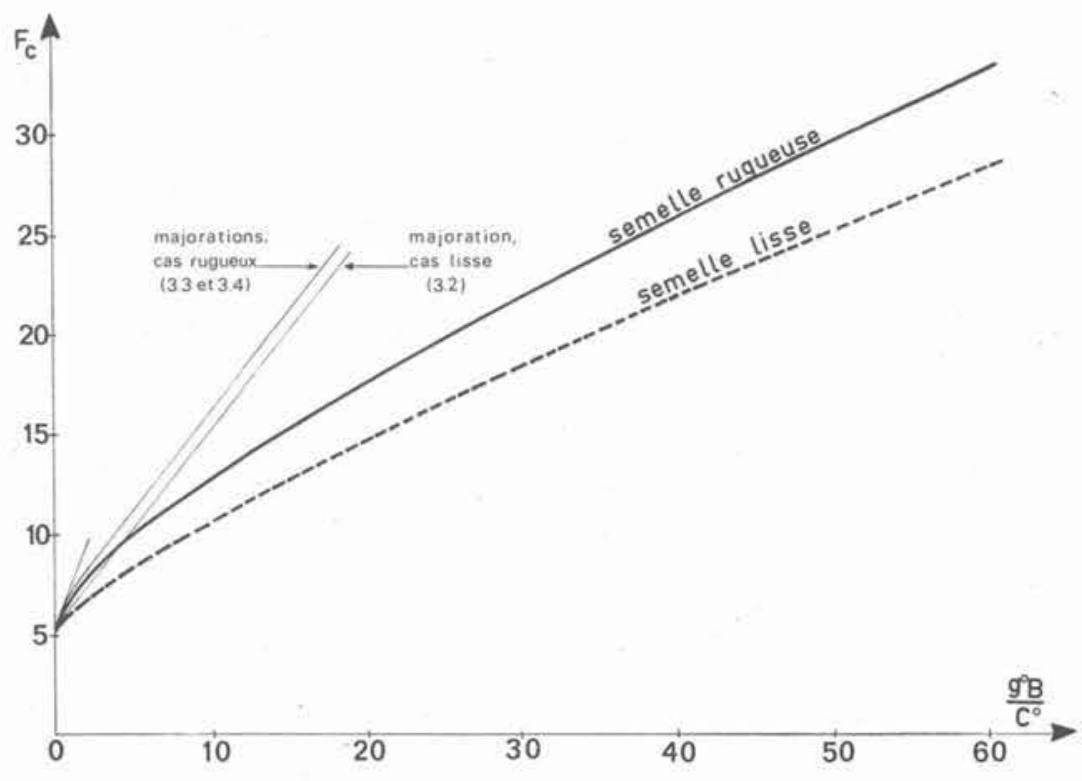

(') Cette formule donne une évaluation de $\mathrm{N}_{c}^{\prime}(\mathrm{B} / h)$ dans le sens de la sécurité. 
solution, incomplète au sens de Bishop, a été complétée statiquement dans les zones non déformées par Davis et Booker [5], et fournit donc la valeur exacte de la capacité portante. Celle-ci se met sous la forme :

$$
p_{\text {uit }}=q+\mathrm{C}^{o} \mathrm{~F}_{c}\left(\frac{g^{o} \mathrm{~B}}{\mathrm{C}^{o}}\right)
$$

où $\mathrm{F}_{c}\left(\frac{g^{o} \mathrm{~B}}{\mathrm{C}^{\circ}}\right)$ est donné par l'abaque de la figure 4 dans le cas d'une semelle "rugueuse " et dans le cas d'une semelle « lisse ». On peut démontrer que les formules (3.2) et (3.3) correspondent à la linéarisation de (3.6) dans le cas de faible non-homogénéité $\left(g^{o} \mathrm{~B} / \mathrm{C}^{o} \# 0\right)$, pour la semelle "lisse » et la semelle " rugueuse " respectivement, confirmant ainsi l'analyse de Spencer [27] par la méthode des perturbations.

Dans la suite, seul le cas de la semelle "rugueuse " étant considéré, nous désignerons par $\mathrm{F}_{c}\left(g^{\circ} \mathrm{B} / \mathrm{C}^{\circ}\right)$ le facteur de capacité portante correspondant sans plus préciser la nature du contact.

\subsection{Solution complète pour $\mathrm{C}^{\circ}=0$}

Lorsque la cohésion en surface est nulle, $\mathrm{C}^{\circ}=0$, la construction de la solution en s'appuyant sur la théorie des équilibres limites plans se révèle impraticable. Des considérations d'analyse dimensionnelle montrent que la capacité portante de la fondation se met alors nécessairement sous la forme :

$$
p_{\text {ult }}=q+\mathrm{K} \mathrm{g}^{o} \mathrm{~B}
$$

où $\mathrm{K}$ est une constante. Une solution complète du problème a été donnée par Salençon [22], fournissant pour $\mathrm{K}$ la valeur exacte : $\mathrm{K}=1 / 4$. D'où la capacité portante pour $\mathrm{C}^{o}=0$ :

$$
p_{\text {ult }}=q+\frac{1}{4} g^{o} \mathrm{~B}
$$

La répartition des contraintes, normales, sous la fondation est alors triangulaire de même pente que le profil de cohésion (fig. 5).

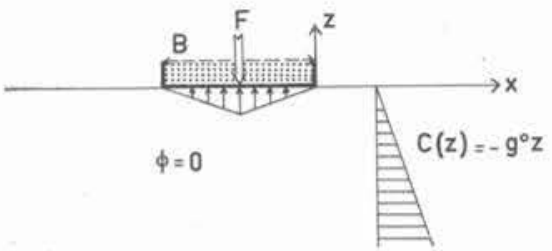

Fig. 5. - Semelle sur sol de cohésion nulle en surface et croissant linéairement avec la profondeur.

\subsection{Comparaison entre solution globale et solution par superposition pour $\mathrm{C}^{\circ} \neq 0$}

Le milieu homogène de cohésion constante égale à $\mathrm{C}^{\circ}$ constitue évidemment un cas particulier du paragraphe 3.2. Pour celui-ci, la valeur bien connue de la capacité portante :

$$
p_{\text {ult }}=q+\mathrm{C}^{\circ}(\pi+2)
$$

est fournie par la solution de Prandtl complétée du point de vue des contraintes dans les zones non déformées, suivant les champs proposés par Shield [28], Bishop [2] ou Sayir et Ziegler [26] par exemple.
La figure 6 représente, à titre d'exemple, le réseau d'écoulement de cette solution avec le prolongement de Shield $\left.{ }^{1}\right)$.

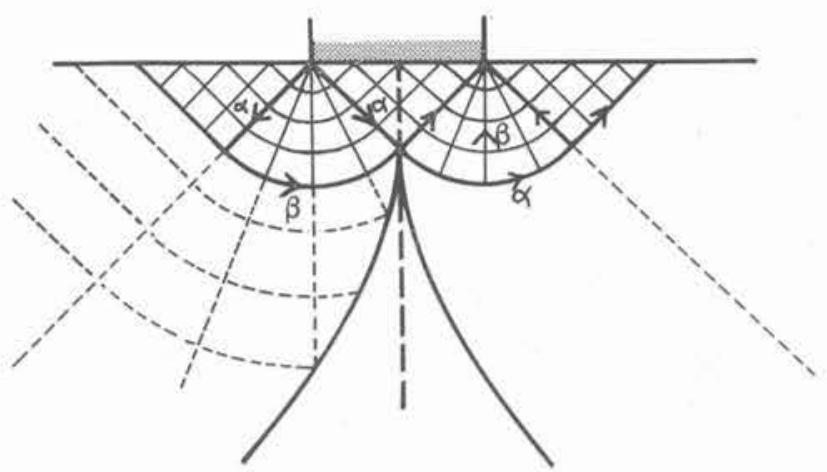

Fig. 6. - Semelle filante sur sol homogène: solution de Prandtl avec le prolongement de Shield.

On dispose ainsi d'un (et même en fait de plusieurs) champ de contrainte $\underline{\sigma}_{c}$ statiquement et plastiquement admissible pour le cas du sol homogène de cohésion $\mathrm{C}^{\circ}$ avec la surcharge $q$, et correspondant à la charge sur la fondation donnée par (3.8).

D'autre part, la solution complète du paragraphe 3.3 fournit, elle, un champ de contraintes statiquement et plastiquement admissible pour le cas du sol de cohésion $\mathrm{C}(z)=-g^{\circ} z$. Ce champ, soit $\underline{\sigma}_{g}$ dans le

\begin{tabular}{|c|c|c|c|}
\hline Région 1 & $\sigma_{z t}=g^{\circ} x$ & $\sigma_{x x}=g^{0} x$ & $\sigma_{t x}=-g^{\circ} z$ \\
\hline Région 2 & $\sigma_{t z}=g^{\circ}\left(2 x+\frac{\mathrm{B}}{2}\right)$ & $\sigma_{x x}=-g^{\circ}\left(2 z+\frac{\mathrm{B}}{2}\right)$ & $\sigma_{2 x}=0$ \\
\hline Région 3 & $\sigma_{z z}=g^{\circ}\left(2 x+\frac{\mathbf{B}}{2}\right)$ & $\sigma_{x x}=0$ & $\sigma_{z x}=0$ \\
\hline Région 4 & $\sigma_{z z}=2 g^{\circ} x$ & $\sigma_{x x}=+2 g^{\circ} z$ & $\sigma_{2 x}=0$ \\
\hline Région 5 & $\sigma_{z z}=2 g^{o} x$ & $\sigma_{x x}=0$ & $\sigma_{t s}=0$ \\
\hline Région 6 & $\sigma_{z z}=0$ & $\sigma_{x x}=+2 g^{0} z$ & $\sigma_{2 x}=0$ \\
\hline Région 7 & $\sigma_{z}=0$ & $\sigma_{u}=0$ & $\sigma_{z x}=0$ \\
\hline
\end{tabular}
cas de surcharge nulle $q=0$, est explicité à la figure 7, et correspond à une pression moyenne sur la fondation égale à $\frac{1}{4} g^{o} \mathrm{~B}$.

(1) Les résultats classiques concernant la théorie des équilibres limites plans seront rappelés au paragraphe 5 au moment de leur utilisation effective. 


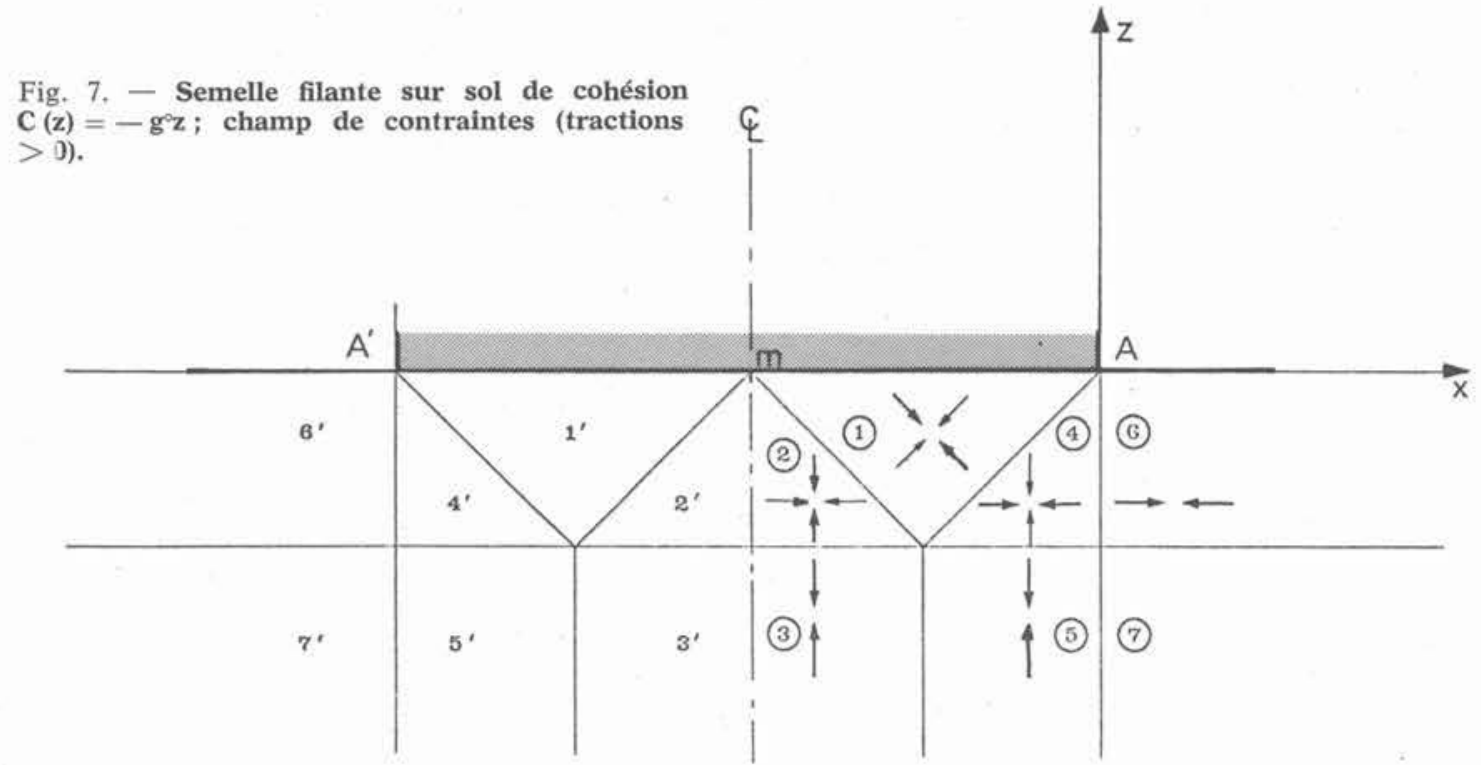

Il est immédiat de vérifier, par exemple en utilisant la représentation de Mohr, que le champ :

$$
\underline{\underline{\sigma}}=\underline{\underline{\sigma}} \underline{\underline{\sigma}} g
$$

obtenu en additionnant en chaque point les contraintes produites par les champs $\underline{\sigma}_{c}$ et $\underline{\sigma}_{g}$, qui est évidemment statiquement admissible, et aussi plastiquement admissible pour le cas du sol de fondation de cohésion (3.1) $\mathrm{C}(z)=\mathrm{C}^{\circ}-\mathrm{g}^{\circ} z$.

On dispose alors ainsi d'une solution statique pour le problème du paragraphe 3.2 ; la valeur correspondante de la pression moyenne sous la fondation soit :

$$
\text { (3.9) (pult) } \text { superp. }_{\text {. }}=q+\mathrm{C}^{\circ}(\pi+2)+\frac{1}{4} g^{o} \mathrm{~B}
$$

est donc un minorant de la capacité portante. C'est bien ce que confirme la comparaison avec les résultats du calcul global exact (formule (3.6) et fig. 4).

La formule linéaire (3.9) peut être interprétée comme correspondant à la superposition des effets de cohésion en surface et de gradient de cohésion. Le fait que la capacité portante réelle soit supérieure à celle donnée par (3.9), traduit l'effet majorateur du couplage entre cohésion en surface et gradient de cohésion.

Pour caractériser cet effet, on peut introduire le coefficient $\mu_{c}$, fonction de $g^{\circ} \mathrm{B} / \mathrm{C}^{o}$ :

$\mu_{c}\left(\frac{g^{o} \mathrm{~B}}{\mathrm{C}^{o}}\right)=\frac{\mathrm{F}_{c}\left(g^{o} \mathrm{~B} / \mathrm{C}^{o}\right)}{(\pi+2)+\frac{1}{4} \frac{g^{o} \mathrm{~B}}{\mathrm{C}^{o}}}=\frac{p_{\mathrm{ult}}-q}{\left(p_{\text {ult }}-q\right)_{\text {superp. }}}$

dont la variation est représentée à la figure 8 (voir Salençon, Florentin et Gabriel [25]).

$\mu_{c}$ est évidemment égal à 1 pour $\mathrm{g}^{\circ} \mathrm{B} / \mathrm{C}^{o}=0\left(^{1}\right)$, et pour $\mathrm{g}^{\circ} \mathrm{B} / \mathrm{C}^{o} \nearrow \infty$. Il passe par un maximum égal à 1.72 pour $g^{\circ} \mathrm{B} / \mathrm{C}^{\circ}=22$.

(1) Sa représentation au voisinage de $g^{\circ} \mathrm{B} / \mathrm{C}^{\circ}=0$ est, par (3.3) et (3.9): $\mu_{c}=1+\frac{7}{4(\pi+2)} \frac{g^{\circ} \mathrm{B}}{\mathrm{C}^{\circ}}$

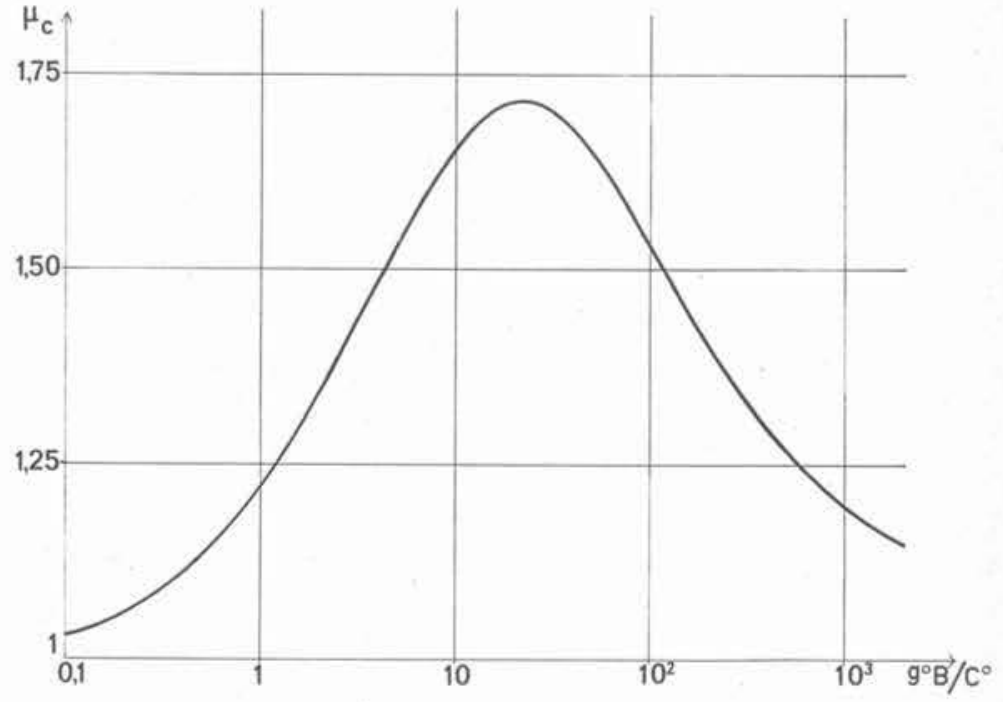

Fig. 8. - Effet majorateur du couplage « $\mathbf{C}^{\circ}-\mathrm{g}^{\circ} \nsim$ : variation de $\mu_{c}$.

\subsection{Cas de la fondation axisymétrique}

La capacité portante d'une fondation superficielle de révolution sur sol $\Phi=0$ illimité dont la cohésion croît linéairement avec la profondeur a été étudiée par Croc, Michel et Pecker [4] en adoptant l'hypothèse de Haar-Karman sur la distribution des contraintes. Pour $\mathrm{C}^{\circ} \neq 0$, la capacité portante se met encore sous la forme (3.6) et le réseau d'écoulement, construit par la méthode des caractéristiques, permet de déterminer le facteur $\mathrm{F}_{c}\left(\mathrm{~g}^{o} \mathrm{~B} / \mathrm{C}^{o}\right)$.

\subsection{Remarque}

Les paragraphes 2 et 3 ci-dessus ont examiné les deux types principaux de non homogénéité : d'une part, par discontinuité dans les propriétés du sol de fondation (la cohésion passe, à l'interface, de la valeur finie C à une valeur infinie pour l'assise rigide), d'autre part, la non-homogénéité continue la plus simple (croissance linéaire de la cohésion avec la profondeur). 
Une liaison entre ces deux types de problèmes de non homogénéité, d'une nature différente de celle qui est l'objet de la présente étude, a été faite par Ostrowska [18] qui a envisagé le cas d'un sol dont la cohésion varie de façon continue mais brutale, passant de la valeur $\mathrm{C}$ à une valeur $\mathrm{C}^{1}$ plus élevée.

Cette étude a été menée par la théorie des équilibres limites plans.

\section{COUCHE DE SOL $\Phi=0$ D'EPAISSEUR LIMITEE ET DE COHESION VARIABLE : ETUDE PAR LA METHODE DE SUPERPOSITION}

\subsection{Présentation}

On se propose maintenant de combiner les deux types de non homogénéité en étudiant, comme cela se présente souvent dans la pratique, la capacité portante d'une semelle filante chargée axialement, sur une couche de sol d'épaisseur limitée $h$, dont la cohésion croît linéairement avec la profondeur.

Comme indiqué au paragraphe 2 , le contact entre la couche de sol de fondation et l'assise rigide est dorénavant supposé s'effectuer avec frottement maximal.

Une première méthode d'étude, ne nécessitant pas de calculs nouveaux par rapport à ceux présentés dans les paragraphes précédents consiste à appliquer la méthode de superposition.

\subsection{Cohésion nulle en surface : $C^{\circ}=0$}

On étudie d'abord le cas où la cohésion du sol de fondation est nulle en surface (fig. 9).

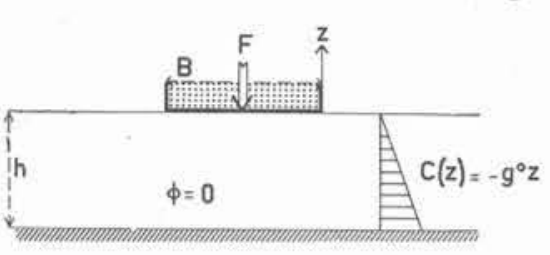

Fig. 9. - Semelle filante sur couche de sol $\Phi=0$ de cohésion nulle en surface et croissant linéairement avec la profondeur.

On obtient immédiatement une solution statique pour le problème en considérant, dans la couche de sol d'épaisseur $h$, la partie correspondante du champ de contraintes, explicité à la figure 7 du cas du sol illimité. En effet, le seul point à vérifier pour s'assurer du caractère plastiquement admissible de ce champ dans le cas présent, se situe au niveau de l'interface : on voit que quel que soit $h$, la contrainte normale $\sigma_{\ldots}$ sur l'interface est compressive; il n'y a donc pas décollement et l'interface étant à frottement maximal, la cission. éventuelle (en région 1 si $h<\mathrm{B} / 4$ ) est admissible. L'addition d'une surcharge compressive $q$, conduit à ajouter une pression isotrope $q$ à l'état de contrainte en tout point et ne modifie pas le raisonnement.

Il s'ensuit que l'on a ainsi une minoration de la capacité portante de la semelle filante dans ce cas :

$$
p_{\text {ult }}=q+\frac{1}{4} g^{o} \mathrm{~B} \text {. }
$$

D'autre part, on sait que dans le cas du sol illimité, le mécanisme d'écoulement associé à la solution statique de la figure 7 , et formant une solution complète, est le mécanisme évanescent, limite quand $\delta$ \} 0 \text { de celui } représenté à la figure 10 , où il y a glissement le long de $m \mathrm{BCD}$ et $m \mathrm{~A}$ (et symétriques) et déformation dans A B C (et symétrique) (voir [22]).

Dans ce mécanisme limite la déformation se produit donc dans une pellicule infinitésimale le long de $\mathrm{m} \mathrm{A}$ (et symétrique). Il est évident que ce mécanisme limite reste valable dans le cas de la couche d'épaisseur limitée $h$, quel que soit $h$; il est associé au champ de contraintes, restreint à la couche, présenté plus haut. On dispose donc d'une solution complète pour le problème et la valeur exacte de la capacité portante est :

$$
p_{\text {ult }}=q+\frac{1}{4} g^{o} \mathrm{~B} \quad \forall h \text { si } \mathrm{C}^{\circ}=0
$$

Ainsi, l'assise rigide n'a aucun effet ${ }^{1}$ ). 
fournit un champ de contrainte statiquement et plastiquement admissible pour le cas

$$
\mathrm{C}(z)=\mathrm{C}^{o}-g^{o} z \text {, épaisseur }=h .
$$

$$
\left(p_{\text {ult }}\right)_{\text {superp. }}=q+\mathrm{C}^{o} \mathrm{~N}^{\prime}\left(\frac{\mathrm{B}}{h}\right)+\frac{1}{4} g^{o} \mathrm{~B}
$$

Ainsi on a la minoration de la capacité portante obtenue par la méthode de superposition :

- pour $\mathrm{B} / h \leqslant \sqrt{2}$ :

$$
\left(p_{\text {ult }}\right)_{\text {superp. }}=q+(\pi+2) \mathrm{C}^{o}+\frac{1}{4} g^{o} \mathrm{~B}
$$

- pour $\mathrm{B} / h \geqslant \sqrt{2}$ :
C'est cette dernière formule que nous conserverons en posant $\mathrm{N}_{c}^{\prime}(\mathrm{B} / h)=\pi+2$ pour $\mathrm{B} / h \leqslant \sqrt{\prime}$.

On voit que comme au paragraphe 3.4 , le fait que la formule (4.3) fournisse une évaluation de la capacité portante dans le sens de la sécurité traduit l'effet majorateur, pour la couche d'épaisseur limitée comme pour le sol illimité, du couplage entre cohésion en surface et gradient de cohésion.

\section{COUCHE DE SOL $\Phi=0$ D'EPAISSEUR LIMITEE ET DE COHESION VARIABLE : CALCUL GLOBAL}

\subsection{Introduction}

On se propose maintenant, comme dans le cas du sol illimité, de procéder au calcul direct global de la capacité portante de la semelle filante sur couche de sol $\Phi=0$ d'épaisseur limitée dont la cohésion croît linéairement avec la profondeur.

La solution exacte de ce problème étant connue pour $\mathrm{C}^{\circ}=0$ (voir paragraphe 4.2 ), il n'y a évidemment plus à considérer que le cas $\mathrm{C}^{\circ} \neq 0$ (cohésion en surface non nulle).

L'étude du problème va s'appuyer sur la théorie des équilibres limites plans pour le matériau de Tresca non homogène, dont nous allons rappeler brièvement les principaux résultats.

\subsection{Equilibres limites plans pour le matériau de Tresca non homogène}

Les équations régissant les problèmes de déformation plane pour le matériau de Tresca non homogène ont été obtenus par Kuznetzov [12]. On pourra également se reporter à Favretti [7], [8] et [9], Olszak, Rychlewski et Urbanowski [17], Salençon [23].

On utilise les notations classiques :

$\sigma_{1}$ et $\sigma_{2}$ sont les contraintes principales dans le plan de la déformation, ici le plan $\mathrm{O} x z$; elles sont ordonnées selon $\sigma_{1} \geqslant \sigma_{2}$, tractions positives ;

On introduit :

$$
\left\{\begin{array}{l}
p=-\left(\sigma_{1}+\sigma_{2}\right) / 2 \\
\theta=\left(\overrightarrow{O x}, \vec{\sigma}_{1}\right)
\end{array}\right.
$$

On sait que dans la zone plastique le problème, pour les contraintes, est hyperbolique quasi-linéaire.

Les lignes caractéristiques sont les lignes de cission maximale, lignes $(\alpha)$ et $(\beta)$ inclinées à $\mp \pi / 4$ sur la direction principale $\sigma_{1}$ (fig. 11).

Les relations le long des caractéristiques, dans le cas d'une cohésion $\mathrm{C}(z)=\mathrm{C}^{\circ}-g^{\circ} z$, s'écrivent :

$$
\begin{array}{lll}
\text { (5.2) } & d p+2 \mathrm{C} d \theta+g^{o} d x=0 & \text { ligne }(\alpha), \\
\text { (5.3) } & d p-2 \mathrm{C} d \theta-g^{o} d x=0 & \text { ligne ( } \beta),
\end{array}
$$

Pour les vitesses, le problème dans les zones plastiques est hyperbolique linéaire; les lignes caractéristiques sont les lignes $(\alpha)$ et $(\beta)$ caractéristiques pour les contraintes; les relations le long des lignes caractéristiques sont les équations de Geiringer, identiques à celles obtenues pour le matériau homogène, qui traduisent la coïncidence des directions principales de la contrainte et de la vitesse de déformation et l'invariance du volume.
Fig. 11. - Matériau de Tresca en déformation plane : caractéristiques des contraintes et des vitesses.

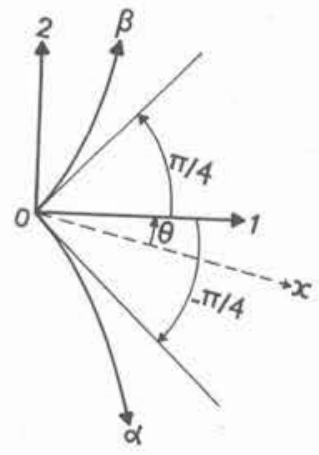

$v_{\alpha}$ et $v_{\beta}$ étant les composantes de la vitesse le long des lignes $(\alpha)$ et $(\beta)$ elles s'écrivent :

$$
\begin{array}{ll}
d v_{\alpha}-v_{\beta} d \theta=0 & \text { ligne }(\alpha), \\
d v_{\beta}+v_{\alpha} d \theta=0 & \text { ligne }(\beta),
\end{array}
$$

De plus, on doit satisfaire la condition de « dissipation positive " ( $\left.{ }^{1}\right)$ :

$$
\frac{\partial v_{\alpha}}{\partial s_{\beta}}-v_{\beta} \frac{\partial \theta}{\partial s_{\beta}}+\frac{\partial v_{\beta}}{\partial s_{\alpha}}+v_{\alpha} \frac{\partial \theta}{\partial s_{\alpha}} \geqslant 0
$$

$\left(\frac{\partial}{\partial s_{\alpha}}\right.$ et $\left.\frac{\partial}{\partial s_{3}}\right)$ désignant les dérivées selon les vecteurs unitaires tangeants aux lignes $(\alpha)$ et $(\beta)$.

Il résulte de ces propriétés que la construction de la solution d'un problème de déformation plane se ramène à celle du réseau de lignes caractéristiques $(\alpha)$ et $(\beta)$ correspondant, à partir des conditions aux limites.

Celui-ci permet ensuite la détermination des contraintes (d'où, par intégration, des efforts) et des vitesses.

\subsection{Construction de la solution}

\subsection{Sol illimité : détail de la solution}

La construction du réseau de lignes caractéristiques pour la couche de sol $\Phi=0$ d'épaisseur limitée et de cohésion linéairement variable s'effectue à partir de la solution du cas du sol illimité et de la même façon qu'on a construit la solution pour la couche de sol $\Phi=0$, homógène, d'épaisseur limitée à partir du cas du sol homogène illimité (paragraphe 2).

(') A proprement parler la condition $\lambda \geqslant 0$ dans la règle d'écoulement écrite sous forme usuelle qui s'identifie, pour le matériau de Tresca, à la posivité de la dissipation. 
La figure 12 représente le réseau de caractéristiques construit par Berthet, Hayot et Salençon dans le cas du sol illimité [1].

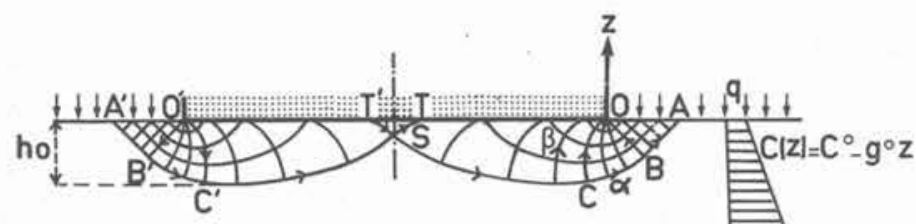

Fig. 12. - Fondation sur sol $\Phi=\mathbf{0}, \mathbf{C}^{\circ} \neq \mathbf{O}, \mathbf{g}^{\circ} \neq \mathbf{0}$, illimité : réseau de caractéristiques.

Ce réseau se compose :

1) d'un champ de Rankine en milieu non homogène, $\mathrm{OAB}$. Les caractéristiques $(\alpha)$ et $(\beta)$ sont des droites.

On a $\theta=\frac{\pi}{2}, p=q+\mathrm{C}^{\circ}-g^{\circ} z$;

2) d'un éventail de Prandtl en milieu non homogène, centré en 0 et d'ouverture $3 \pi / 4$ : OBC. OB est la caractéristique $(\beta)$ rectiligne le long de laquelle l'éventail se raccorde au champ de Rankine ; OC est la caractéristique $(\beta)$, curviligne, issue de $O$ et tangente à $\mathrm{O}^{\prime} \mathrm{O}$, base de la semelle, en conséquence de l'hypothèse de frottement maximal sous la fondation ;

3) la construction du réseau est ensuite poursuivie par les caractéristiques $(\alpha)$ s'appuyant sur OC et les caractéristiques $(\beta)$ tangentes à $\mathrm{OO}^{\prime}$. Le réseau est symétrique par rapport à l'axe de la fondation et est limité par les caractéristiques SCBA $(\alpha)$ et ST $(\beta)$ (et symétriques) qui se coupent sur l'axe de symétrie en un point où $\theta=0$ : en effet par raison de symétrie, l'axe de la fondation est nécessairement direction principale pour les contraintes ; cette condition fixe la longueur $\mathrm{OA}$, et les autres dimensions du réseau, inconnues a priori.

En particulier ce réseau s'enfonce dans le sol jusqu'à la profondeur $h_{o}$. La variation de $h_{o} / \mathrm{B}$ en fonction du facteur sans dimension $g^{\circ} \mathrm{B} / \mathrm{C}^{\circ}$ a été donnée dans [1] : $h_{o} / \mathrm{B}$ est une fonction décroissante de $g^{o} \mathrm{~B} / \mathrm{C}^{o}$ (fig. 13) depuis : $h_{o} / \mathrm{B}=\sqrt{2} / 2$ pour $g^{\circ} \mathrm{B} / \mathrm{C}^{\circ}=0$ jusqu'à $h_{o} / \mathrm{B} \searrow 0$ pour $g^{\circ} \mathrm{B} / \mathrm{C}^{\circ} \not \infty$ (voir paragraphe 4.2).

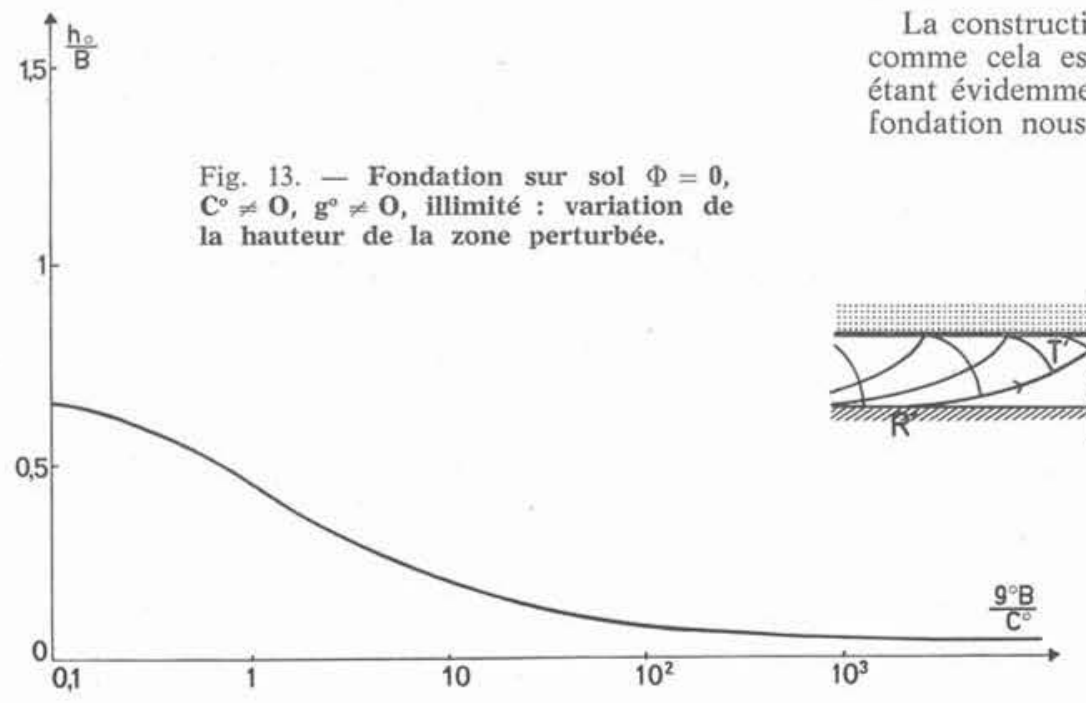

\subsection{Couche de sol d'épaisseur $h \geqslant h_{o}$}

On voit alors que si l'on a affaire à une couche de sol d'épaisseur limitée $h \geqslant h_{o}$, la construction du réseau de caractéristiques de la fig. 12 peut être reproduite sans changement (fig. 14).

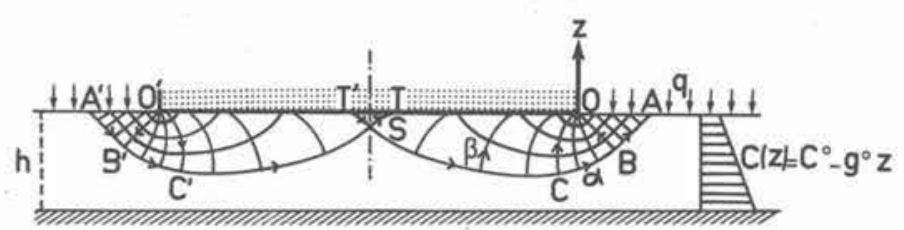

Fig. 14. - Fondation sur couche de sol $\Phi=\mathbf{0}, \mathbf{C}^{\circ} \neq \mathbf{0}$, $\mathbf{g}^{\circ} \neq \mathbf{O}$, d'épaisseur $\mathbf{h} \geqslant \mathbf{h}_{0}$.

L'écoulement plastique du sol sous la fondation se fait suivant le champ de vitesses construit par Berthet, Hayot et Salençon dans ce réseau de lignes : on a donc encore affaire à une solution incomplète (de type cinématique). D'autre part le prolongement du champ de contraintes donné par Davis et Booker peut être conservé, en conséquence de l'hypothèse de frottement maximal à l'interface entre couche de sol et assise rigide.

Dans le triangle STT' le prolongement du champ de contraintes par le champ en équilibre et à la limite d'écoulement défini par les conditions aux limites sur ST et ST' $^{\prime}$ est admissible : la cission sur TT' y est évidemment inférieure ou égale à la cission maximale et les contraintes sont compressives. La solution est donc complète.

En conclusion : à $g^{\circ} \mathrm{B} / \mathrm{C}^{\circ}$ donné, si $h \geqslant h_{0}$ il n'y a pas d'influence de l'assise rigide et la capacité portante exacte de la semelle filante est :

$$
p_{\text {ult }}=q+\mathrm{C}^{o} \mathrm{~F}_{c}\left(\frac{g^{o} \mathrm{~B}}{\mathrm{C}^{o}}\right) \quad \text { si } h / \mathrm{B} \geqslant h_{o} / \mathrm{B}
$$

\subsection{Couche de sol d'épaisseur $h \leqslant h_{o}$}

Si l'épaisseur de la couche est inférieure à $h_{o}$, le réseau de caractéristiques de la figure 12 n'est plus utilisable : la présence de l'assise rigide se fait alors sentir.

La construction de la solution s'effectue dans ce cas, cela est représenté à la figure 15 . Le réseau étant évidemment symétrique par rapport à l'axe de la fondation nous n'en décrirons que la moitié :

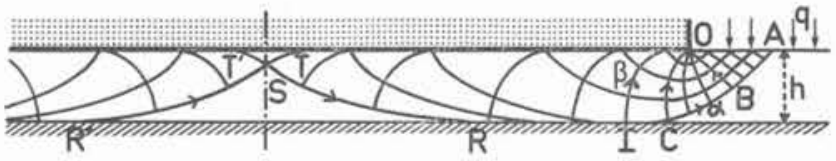

Fig. 15. - Fondation sur couche de sol $\Phi=0, \mathbf{C}^{\circ} \neq \mathbf{0}, \mathrm{g}^{\circ} \neq \mathbf{0}$, d'épaisseur $h \leqslant h_{0}$ : réseau de caractéristiques. 
1) $\mathrm{OAB}$ est un champ de Rankine en milieu non homogène : $\theta=\pi / 2, p=q+\mathrm{C}^{\circ}-g^{\circ} z$;

2) $\mathrm{OBC}$ un éventail de Prandtl en milieu non homogène, centré en $\mathrm{O}$ et d'ouverture $3 \pi / 4$;

3) la longueur de $O A$, qui fixe les dimensions des réseaux $\mathrm{OAB}$ et $\mathrm{OBC}$ est déterminée par la condition de tangence de la caractéristique $(\alpha)$ CBA avec l'interface sol-assise rigide ; $\mathrm{OA}=a$ est une fonction de $h$, de $g^{o}$ et de $\mathrm{C}^{\circ}$, nécessairement de la forme :

$$
a=h \varphi\left(g^{\circ} h / \mathrm{C}^{\circ}\right)
$$

mais comme on le verra dans la description de la procédure pratique de construction de la solution, la détermination de cette fonction ne se révèle pas utile ;

4) la construction du réseau au-delà de ces champs (identiques aux champs homologues dans le cas du sol illimité) se poursuit, à partir de $\mathrm{OC}$ et $\mathrm{Cl}$ en s'appuyant sur les conditions aux limites :

a) à l'interface sous la fondation, le frottement est maximal, les caractéristiques ( $\beta$ ) y sont tangentes ;

b) à l'interface entre le sol et l'assise rigide, le frottement est maximal et les caractéristiques $(\alpha)$ y sont tangentes;

On a affaire à des problèmes de Goursat et de Riemann dont la résolution pas à pas en suivant les caractéristiques est classique.

Le réseau est limité par les caractéristiques SR $(\alpha)$ et ST ( $\beta$ ) qui se coupent sur l'axe de la fondation au point $\mathrm{S}$ où $\theta=0$. Il est ensuite complété par symétrie.

Du point de vue des contraintes, le champ peut être complété sans difficulté dans la couche de sol à droite de IBA, dans STT' et SRR' par des champs en équilibre et à la limite d'écoulement (fig. 16) :

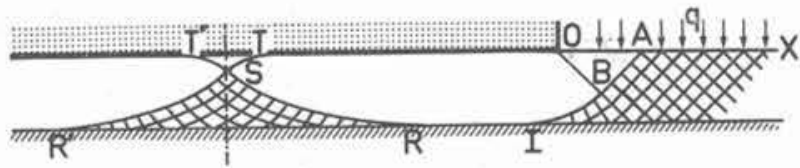

Fig. 16. - Fondation sur couche de sol $\Phi=\mathbf{0}, \mathbf{C}^{\circ} \neq \mathbf{0}$, $\mathrm{g}^{\circ} \neq \mathbf{O}$, d'épaisseur $\mathrm{h} \leqslant \mathrm{h}_{\mathrm{o}}$ : prolongement du champ de contraintes.

1) à droite de IBA, le champ défini par les conditions sur $\mathrm{O} x$ et IBA, qui fournit des contraintes compressives sur l'interface, et une cission évidemment inférieure à la cission maximale ;

2) dans STT' : le champ défini par les conditions sur ST et $\mathrm{ST}^{\prime}$ comme pour le sol illimité ;

3) dans SRR' : le champ défini par les conditions sur $S R$ et $S^{\prime}$ ' qui fournit également des contraintes compressives à l'interface et une cission inférieure à la cission maximale.

La solution est donc statique.

$\mathrm{Du}$ point de vue cinématique, le champ de vitesses peut être construit par les équations de Geiringer (5.4) et (5.5) en respectant (5.6); il correspond à un mode de déformation dans lequel STT' est rigide et s'enfonce verticalement comme la fondation, SRR' est rigide immobile, il y a discontinuité de vitesse suivant les caractéristiques ST, SR et IBA et glissement le long de TO et RI, et il y a déformation dans STOABIRS (et symétriques).

La solution est donc complète et fournit la valeur exacte de la capacité portante.

Remarque : incidemment, on notera que la mise en évidence de la solution complète ci-dessus pour $h<h_{o}$, démontre que la solution complète présentée au paragraphe 5.31 pour le sol illimité est certainement celle qui correspond au minimum de l'épaisseur perturbée (rappelons en effet que les théorèmes d'unicité concernant les solutions complètes d'écoulement plastique libre ne sont que partiels).

\subsection{Construction pratique}

La construction du réseau est effectuée numérique ment en intégrant pas à pas les équations (5.2) et (5.3) le long des lignes caractéristiques.

Afin de limiter le nombre de calculs on procède à une analyse préalable du problème : la surcharge $q$ n'intervenant que de façon additive, les calculs seront effectués pour $q=0$; l'analyse dimensionnelle montre que la solution ne dépend que de deux paramètres sans dimensions, par exemple $\mathrm{B} / h$ et $g^{\circ} \mathrm{B} / \mathrm{C}^{o}$ ou $\mathrm{B} / h$ et $g^{\circ} h / \mathrm{C}^{\circ}$, tandis que la capacité portante $p_{\text {ul }}$ sera à rapporter à une contrainte de référence, soit $\mathrm{C}^{\circ}$ par exemple.

Comme on le verra dans la suite, le premier choix de paramètres se révèle bon pour la présentation et la discussion des résultats obtenus; ce sont d'ailleurs ces paramètres qui interviennent dans les deux problèmes de base étudiés plus haut : couche de sol homogène d'épaisseur limitée d'une part, sol illimité de cohésion variable d'autre part.

Par contre, le deuxième groupe de paramètres correspond à la façon effective dont est mise en œuvre la procédure de calcul numérique.

On travaille en effet à $g^{\circ} h / \mathrm{C}^{\circ}$ fixé ; dans la représentation graphique cela signifie que le profil de cohésion est fixé ainsi que l'épaisseur de la couche. On fait varier $\mathrm{B} / h$, c'est-à-dire que, graphiquement, on fait varier la largeur de la fondation.

Partant de $\mathrm{B} / h=0$, on fait croître $\mathrm{B} / h$. On est d'abord dans le cas où l'assise rigide n'a pas d'influence, jusqu'à ce que B atteigne la valeur critique pour laquelle $h$ est l'épaisseur de la zone perturbée correspondant à $g^{o} \mathrm{~B} / \mathrm{C}^{\circ}$ (fig. 13).

Pour l'étude au-delà de cette valeur critique de $B$, on construit le réseau de la figure 15: on part des données sur $\mathrm{OB}$ ( $p$ et $\theta$ connus du champ de Rankine) en progressant le long des caractéristiques $(\alpha)$ que l'on construit une à une autour de $\mathrm{O}$, jusqu'à la caractéristique $(\alpha)$ tangente à l'interface fixé à la profondeur $h$. On poursuit ensuite la construction de façon systématique en progressant encore le long des lignes $(\alpha)$, à partir de leur point de tangence sur l'interface solassise rigide. On obtient ainsi le réseau indéfini vers la gauche (fig. 17), avec sur chaque caractéristique $(\alpha)$ construite, un point où $\theta=0$. Ce point représente le point S (fig. 15) pour la fondation dont l'axe passerait par lui. On explore ainsi, à $g^{\circ} h / \mathrm{C}^{\circ}$ fixé, toutes les valeurs de $\mathrm{B} / h$ supérieures à la valeur critique.
Fig. 17. - Construction du réseau indéfini à $\mathrm{g}^{\circ} \mathrm{h} / \mathrm{C}^{\circ}$ fixé $(1,386)$.

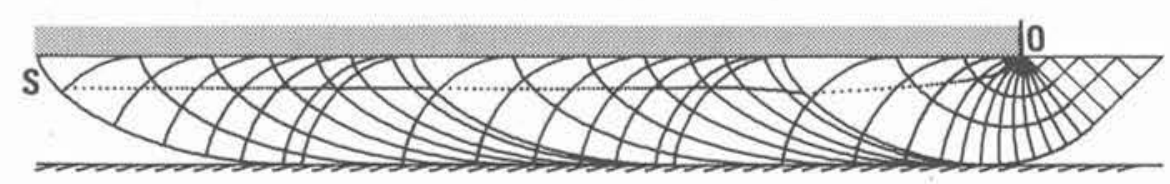


La construction du réseau fournit en même temps les valeurs de $p$ et $\theta$ aux nœuds et donc le champ de contraintes.

Pour le calcul de $p_{\text {ult }}$, il suffit d'intégrer les contraintes connues sur STO et d'en prendre la composante verticale qui représente, au signe près, $p_{\text {ult }} \times \mathrm{B} / 2$. En fait, dans la pratique, il est plus commode d'intégrer la contrainte principale $\sigma_{2}$, depuis $\mathrm{O}$ jusqu'à $\mathrm{S}$ situé sur l'axe de fondation, en suivant la ligne (S) lieu des points $\mathrm{S}$ situés sur chaque ligne $(\alpha)$ (fig. 17 ), d'où avec les conventions de signes :

$$
p_{\text {ult }}=\frac{2}{\mathrm{~B}} \int_{\operatorname{selon}(\mathrm{S}) .}^{\mathrm{S}} \sigma_{2} d x
$$

\section{RESULTATS DU CALCUL GLOBAL}

\subsection{Choix des paramètres}

Comme on 1'a indiqué en 5.34 , la formule finale donnant $p_{\text {ult }}$ par le calcul global, est nécessairement de la forme :

$$
p_{\text {ult }}=q+\mathrm{C}^{o} F_{c}\left(\frac{\mathrm{B}}{h}, \frac{g^{o} \mathrm{~B}}{\mathrm{C}^{o}}\right)
$$

ou de façon équivalente :

$$
p_{\mathrm{ult}}=q+\mathrm{C}^{o} G_{c}\left(\frac{\mathrm{B}}{h}, \frac{g^{o} h}{\mathrm{C}^{o}}\right)
$$

L'étude détaillée des divers types de représentations a été présentée par Matar [16] et nous nous restreindrons, pour simplifier l'exposé, à la plus commode d'entre elles du point de vue pratique.

Comme dans le cas du milieu illimité il est utile, pour mettre en évidence l'effet majorateur du couplage cohésion en surface-gradient de cohésion, d'introduire le rapport $\mu_{c}$ :

$$
\mu_{c}=\frac{p_{\text {ult }}-q}{\left(p_{\text {ult }}-q\right)_{\text {superp. }}}
$$

où $\left(p_{\text {ult }}-q\right)_{\text {superp. }}$ est donnée par (4.3) que nous rappelons :

$$
\left(p_{\text {ult }}-q\right)_{\text {superp. }}=\mathrm{C}^{o} \mathrm{~N}_{c}^{\prime}\left(\frac{\mathrm{B}}{h}\right)+\frac{1}{4} g^{o} \mathrm{~B}
$$

Nous étudierons les variations de $\mu_{c}$ en fonction de $\mathrm{B} / h$ et $g^{o} \mathrm{~B} / \mathrm{C}^{o}$.

On a démontré au paragraphe 4.3 que $\mu_{c}\left(\frac{\mathrm{B}}{h}, \frac{g^{o} \mathrm{~B}}{\mathrm{C}^{o}}\right) \geqslant 1$; on obtient sans difficulté une majoration de $\mu_{c}$ en remarquant que la capacité portante de la fondation est nécessairement inférieure (voir par exemple [24]) à celle obtenue dans le cas d'une couche homogène de même épaisseur dont la cohésion serait égale à $\mathrm{C}^{o}+g^{\circ} h$; d'où :

$$
p_{\text {ult }}-q \leqslant\left(\mathrm{C}^{o}+g^{o} h\right) \mathrm{N}_{c}^{\prime}(\mathrm{B} / h)
$$

et :

(6.4)

$$
1 \leqslant \mu_{c}\left(\frac{\mathrm{B}}{h}, \frac{g^{\circ} \mathrm{B}}{\mathrm{C}^{o}}\right) \leqslant \frac{1+\frac{g^{\circ} \mathrm{B}}{\mathrm{C}^{o}} \times\left(\frac{\mathrm{B}}{h}\right)^{-1}}{1+\frac{1}{4} \frac{g^{o} \mathrm{~B}}{\mathrm{C}^{o}}\left[\mathrm{~N}_{c}^{\prime}\left(\frac{\mathrm{B}}{h}\right)\right]^{-1}}
$$

\subsection{Propriétés de $F_{c}$ et $\mu_{c}$}

\subsection{Non influence de lassise rigide}

$\mathrm{A} g^{\circ} \mathrm{B} / \mathrm{C}^{o}$ fixé, si $\mathrm{B} / h$ est inférieur à la valeur critique donnée par la figure 13 , soit $(\mathrm{B} / h)_{o}$, l'assise rigide n'a pas d'influence sur la valeur de la capacité portante.
Autrement dit on a :

$$
\left\{\begin{array}{l}
\text { si } 0 \leqslant \mathrm{~B} / h \leqslant(\mathrm{~B} / h)_{o}, \\
\text { alors } F_{c}\left(\frac{\mathrm{B}}{h}, \frac{g^{o} \mathrm{~B}}{\mathrm{C}^{o}}\right) \equiv \mathrm{F}_{c}\left(\frac{g^{o} \mathrm{~B}}{\mathrm{C}^{o}}\right) .
\end{array}\right.
$$

Comme indiqué par la figure $13,(\mathrm{~B} / h)_{0}$ est une fonction croissante de $\mathrm{g}^{\circ} \mathrm{B} / \mathrm{C}^{\circ}$, qui part de la valeur $\sqrt{2}$ pour $g^{o} \mathrm{~B} / \mathrm{C}^{\circ}=0$, et tend vers l'infini quand $g^{\circ} \mathrm{B} / \mathrm{C}^{o} \nearrow \infty$.

II résulte alors de (4.3), où $\mathrm{N}_{c}^{\prime}\left(\frac{\mathrm{B}}{h}\right)=\pi+2$ si $\mathrm{B} / h \leqslant \sqrt{2}$, que :

$$
\left\{\begin{array}{l}
\text { si } 0 \leqslant \mathrm{~B} / h \leqslant \sqrt{2}, \\
\text { alors } \mu_{c}\left(\frac{\mathrm{B}}{h}, \frac{g^{o} \mathrm{~B}}{\mathrm{C}^{o}}\right) \equiv \mu_{c}\left(\frac{g^{o} \mathrm{~B}}{\mathrm{C}^{o}}\right) ;
\end{array}\right.
$$

par contre $\mu_{c}$ dépend explicitement de $\mathrm{B} / h$ dès que $\mathrm{B} / h$ est supérieur à $\sqrt{2}$.

\subsection{2. $S i g^{\circ} \mathrm{B} / \mathrm{C}^{\circ} \searrow 0$}

Pour $g^{\circ} \mathrm{B} / \mathrm{C}^{\circ}=0$ on a évidemment :

d'où :

$$
F_{c}\left(\frac{\mathrm{B}}{h}, \frac{g^{o} \mathrm{~B}}{\mathrm{C}^{o}}\right)=F_{c}\left(\frac{\mathrm{B}}{h}, 0\right) \equiv \mathrm{N}_{c}^{*}\left(\frac{\mathrm{B}}{h}\right),
$$

$$
\mu_{c}\left(\frac{\mathrm{B}}{h}, 0\right)=1 \quad \forall \mathrm{B} / h
$$

La continuité quand $g^{\circ} \mathrm{B} / \mathrm{C}^{\circ} \searrow 0$ est assurée par l'encadrement (6.4).

\subsection{3. $S i g^{\circ} \mathrm{B} / \mathrm{C}^{\circ} \nearrow \infty$}

Pour $\mathrm{C}^{o}=0$ on a d'après $(4.1)$ :

$$
p_{\text {ult }}-q=\frac{1}{4} g^{\circ} \mathrm{B} \forall \mathrm{B} / h
$$

d'où :

$$
\mu_{c}\left(\frac{\mathrm{B}}{h}, \infty\right)=1, \forall \mathrm{B} / h .
$$

Quand $g^{o} \mathrm{~B} / \mathrm{C}^{\circ} \nearrow \infty$, on a $(\mathrm{B} / h)_{0} \nearrow \infty$. C'est-à-dire que quand $g^{n} \mathrm{~B} / \mathrm{C}^{o} \nearrow \infty, F_{c}\left(\frac{\mathrm{B}}{h}, \frac{g^{o} \mathrm{~B}}{\mathrm{C}^{o}}\right)$ s'identifie à $\mathrm{F}_{c}\left(\frac{g^{o} \mathrm{~B}}{\mathrm{C}^{o}}\right), \forall \mathrm{B} / h$.

Il en résulte alors, puisque $\mathrm{N}_{c}^{\prime}\left(\frac{\mathrm{B}}{h}\right) \geqslant \mathrm{N}_{c}=\pi+2$, que si $g^{\circ} \mathrm{B} / \mathrm{C}^{\circ} \nearrow \infty$,

alors $1 \leqslant \mu_{c}\left(\frac{\mathrm{B}}{h}, \frac{g^{o} \mathrm{~B}}{\mathrm{C}^{o}}\right) \leqslant \mu_{c}\left(\frac{g^{o} \mathrm{~B}}{\mathrm{C}^{o}}\right), \forall \mathrm{B} / h$. 


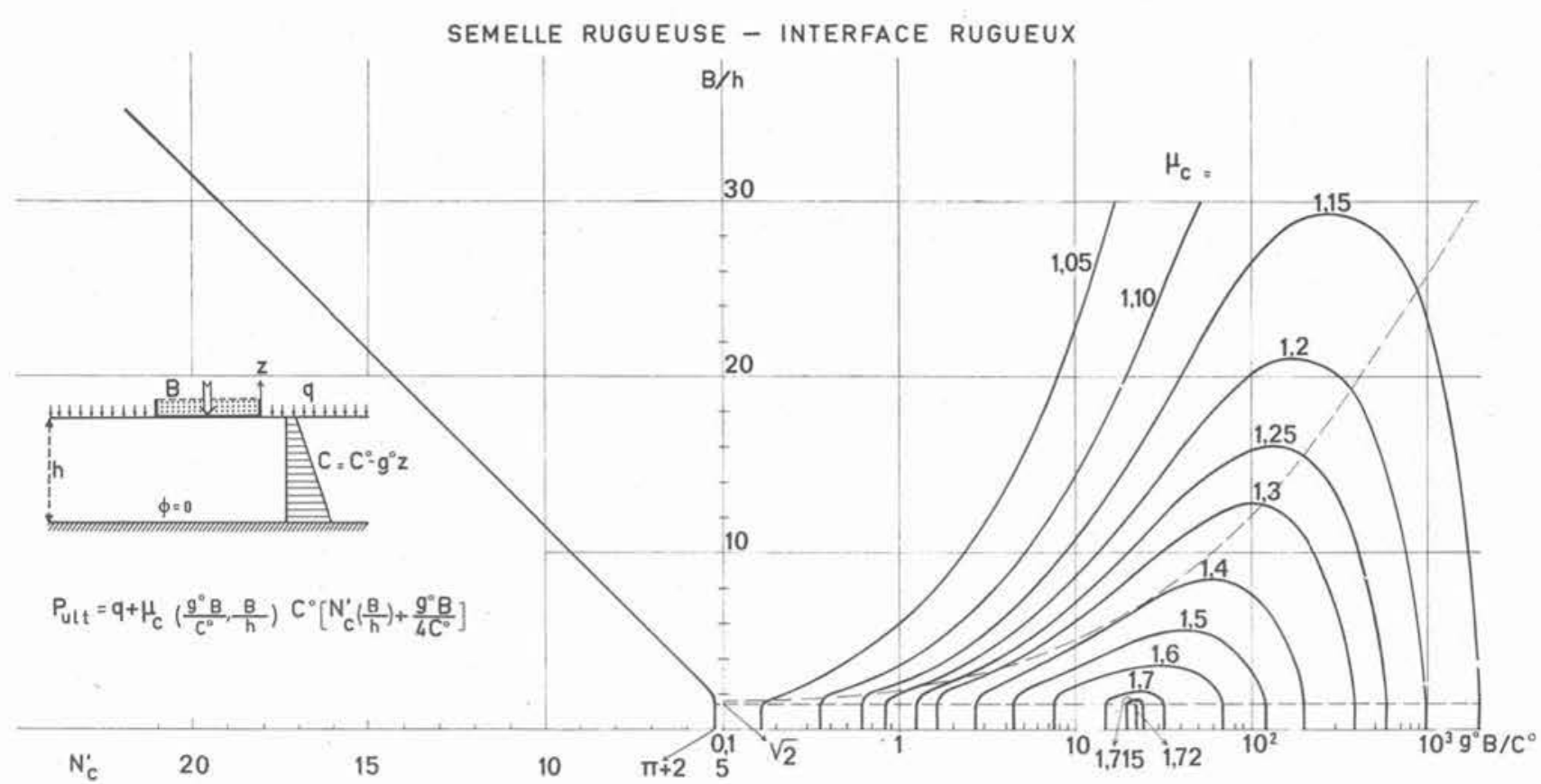

Fig. 18. - Courbes " iso $-\mu_{6} n$.

Les calculs numériques antérieurs effectués pour $\mu_{c}\left(\frac{g^{o} \mathrm{~B}}{\mathrm{C}^{o}}\right)$ ont montré que :

si $\frac{g^{o} \mathrm{~B}}{\mathrm{C}^{\circ}} \nearrow \infty$

alors $\mu_{c}\left(\frac{g^{o} \mathrm{~B}}{\mathrm{C}^{o}}\right) \searrow 1$,

il s'ensuit que

$$
\left\{\begin{array}{l}
\text { si } \frac{g^{o} \mathrm{~B}}{\mathrm{C}^{o}} \nearrow \infty \\
\text { alors } \mu_{c}\left(\frac{\mathrm{B}}{h}, \frac{g^{o} \mathrm{~B}}{\mathrm{C}^{o}}\right) \searrow 1, \forall \mathrm{B} / h .
\end{array}\right.
$$

\subsection{Si B/h $₫ \infty$}

La formule (2.2) fournit l'expression asymptotique de $\mathrm{N}_{c}^{\prime}\left(\frac{\mathrm{B}}{h}\right)$ quand $\mathrm{B} / h \pi \infty$.

$$
\mathrm{N}_{c}^{\prime}\left(\frac{\mathrm{B}}{h}\right)=\pi+1+\frac{1}{2} \mathrm{~B} / h .
$$

Il résulte alors de l'encadrement (6.4) que

$$
\left\{\begin{array}{l}
\text { si } \mathrm{B} / \mathrm{H} / \infty \\
\text { alors } \mu_{c}\left(\frac{\mathrm{B}}{h}, \frac{g^{o} \mathrm{~B}}{\mathrm{C}^{o}}\right) \searrow 1 \forall g^{\circ} \mathrm{B} / \mathrm{C}^{\circ} \geqslant 0 .
\end{array}\right.
$$

\subsection{Représentation graphique}

La représentation graphique la plus commode des résultats obtenus est donnée à la figure 18, par des courbes « iso $-\mu_{c}$ " dans le plan « $\frac{g^{\circ} \mathrm{B}}{\mathrm{C}^{\circ}}, \frac{\mathrm{B}}{h}$ ».

Le plan est séparé en deux régions par la courbe donnant $(\mathrm{B} / h)_{o}$ en fonction de $\frac{g^{o} \mathrm{~B}}{\mathrm{C}^{o}}$ : au-dessous de cette courbe, la présence de l'assise rigide n'intervient pas dans la valeur de la capacité portante de la fondation.

En regard de ce plan, on a tracé la courbe donnant $\mathrm{N}^{\prime}{ }_{c}\left(\frac{\mathrm{B}}{h}\right)$ en fonction de $\mathrm{B} / h$.

L'abaque ainsi obtenu est d'un emploi commode pour les calculs pratiques. On applique la formule :

$$
p_{\text {ult }}=q+\mu_{c} \mathrm{C}^{o} \times\left(\mathrm{N}_{c}^{\prime}+\frac{1}{4} \frac{g^{o} \mathrm{~B}}{\mathrm{C}^{\circ}}\right)
$$

où les valeurs des coefficients $\mathrm{N}_{c}^{\prime}$ et $\mu_{c}$ sont lues directement ou interpolées.

\section{COMMENTAIRES SUR LES RESULTATS}

On voit que $\mu_{c}\left(\frac{\mathrm{B}}{h}, \frac{g^{o} \mathrm{~B}}{\mathrm{C}^{o}}\right)$ est une fonction décroissante de $\frac{\mathrm{B}}{h}, \forall g^{o} \mathrm{~B} / \mathrm{C}^{\circ}$. Le maximum étant égal à $\mu_{c}\left(\frac{g^{o} \mathrm{~B}}{\mathrm{C}^{o}}\right)$ il s'ensuit que l'on a toujours :

$$
p_{\text {ult }} \leqslant q+\mu_{c}\left(\frac{g^{o} \mathrm{~B}}{\mathrm{C}^{o}}\right) \mathrm{C}^{o}\left(\mathrm{~N}_{c}^{\prime}\left(\frac{\mathrm{B}}{h}\right)+\frac{1}{4} \frac{g^{o} \mathrm{~B}}{\mathrm{C}^{o}}\right)
$$

l'inégalité étant stricte dès que $\mathrm{B} / h>\sqrt{ } 2$, pour $g^{\circ} \mathrm{B} / \mathrm{C}^{\circ}>0$.
Ceci montre que les effets majorateurs dus à la présence de l'assise rigide pour un sol homogène d'une part, et au couplage entre cohésion en surface et gradient de cohésion pour un sol illimité d'autre part, ne se cumulent pas.

Ainsi, la quatrième méthode envisagée au paragraphe 1 pour traiter le problème conduirait à une surestimation de la capacité portante d'autant plus importante que $\mathrm{B} / h$ est plus grand. Elle ne peut donc être retenue.

La troisième méthode envisagée au paragraphe 1 , proposait de traiter la couche comme homogène avec une 
cohésion moyenne, qui pourrait être la moyenne de la cohésion dans la couche (c'est-à-dire la cohésion à la demi-profondeur). La formule correspondante pour $p_{\text {ult }}$ serait alors : :

$$
\bar{p}_{\text {ult }}=q+\mathrm{N}_{c}^{\prime}\left(\frac{\mathrm{B}}{h}\right) \quad \mathrm{C}^{o}\left(1+\frac{g^{o} \mathrm{~B}}{2 \mathrm{C}^{\circ}} /\left(\frac{\mathrm{B}}{h}\right)\right)
$$

Les calculs effectués dans plusieurs cas, tels que les deux exemples typiques présentés au paragraphe 8 , montrent que :

- Dans le cas où $\mathrm{B} / h<(\mathrm{B} / h)_{o}$, la formule (7.2) conduit à une surestimation de la capacité portante d'autant plus importante que $g^{o} \mathrm{~B} / \mathrm{C}^{o}$ est grand; ceci se comprend si l'on remarque que, dans ce cas, la zone plastique qui contribue au calcul de la capacité portante (zone déformée) n'intéresse pas toute la couche.

- Dans le cas où $\mathrm{B} / h>(\mathrm{B} / h)_{o}$, les résultats obtenus par le calcul global et par la formule approchée
(7.2) sont plus voisins; la concordance se révèle même excellente lorsque le point représentatif du cas étudié dans le plan " $g^{\circ} \mathrm{B} / \mathrm{C}^{\circ}, \mathrm{B} / h$ » est éloigné de la courbe séparatrice donnant $(\mathrm{B} / h)_{o}$.

On disposerait donc dans ce cas d'une méthode de calcul approchée simplifiée, mais ses conditions d'utilisation en limitent beaucoup l'intérêt. Elle nécessite en effet de disposer de la courbe donnant $(\mathrm{B} / h)_{0}$ en fonction de $g^{o} \mathrm{~B} / \mathrm{C}^{o}$ (la courbe donnant $\mathrm{N}_{c}^{\prime}(\mathrm{B} / h)$ pouvant être remplacée par l'utilisation de la formule approchée (2.2) qui va dans le sens de la sécurité); de plus, pour $\mathrm{B} / h<(\mathrm{B} / h)_{o}$ on doit se reporter au calcul global.

Compte-tenu de sa facilité d'emploi, il paraît donc préférable d'avoir recours, dans tous les cas, à l'abaque de la figure 18 , qui a de plus l'avantage de donner de façon sûre la capacité portante globale.

\section{EXEMPLES DE CALCUL}

\subsection{Premier exemple}

Fondation de largeur

Surcharge nulle

Couche d'épaisseur

$B=40 \mathrm{~m}$

$q=0$

$h=10 \mathrm{~m}$

$\mathrm{C}^{o}=10^{3} \mathrm{~Pa}\left(\simeq 0.1 \mathrm{t} / \mathrm{m}^{2}\right)$

Cohésion en surface

Gradient vertical

descendant

de cohésion

$$
\begin{aligned}
& g^{\circ}=2.5 \times 10^{3} \mathrm{~N} / \mathrm{m}^{3} \\
&\left(\simeq 0.25 \mathrm{t} / \mathrm{m}^{3}\right)
\end{aligned}
$$

On a alors

$$
g^{o} \mathrm{~B} / \mathrm{C}^{o}=10^{2} \text { et } \mathrm{B} / h=4
$$

En se reportant à l'abaque de la figure 18 , on remarque que dans ce cas on a $\mathrm{B} / h<(\mathrm{B} / h)_{o}=12$, et on lit :

$$
\mu_{c}=1.48
$$

$\mathrm{N}^{\prime}{ }_{c}=6.25$ (la formule $(2.2)$ donne $\mathrm{N}^{\prime}{ }_{c}=6.14$ )

d'où par application de

$$
p_{\text {ult }}=\mu_{c} \mathrm{C}^{o}\left(\mathrm{~N}_{c}^{\prime}+\frac{1}{4} \frac{\mathrm{g}^{o} \mathrm{~B}}{\mathrm{C}^{o}}\right)
$$

il vient :

soit :

$$
p_{\text {ult }}=1.48 \times 10^{3} \times(6.25+25) \mathrm{Pa}
$$

$$
p_{\text {ult }}=4.62 \times 10^{4} \mathrm{~Pa}\left(=4.71 \mathrm{t} / \mathrm{m}^{2}\right)
$$

Par la formule (7.2) on obtiendrait :

d'où :

$$
\bar{p}_{\text {ult }}=6.25 \times 10^{3} \times(1+50 / 4) \mathrm{Pa}
$$

$$
\bar{p}_{\text {ult }}=8.44 \times 10^{4} \mathrm{~Pa} \quad\left(=8.61 \mathrm{t} / \mathrm{m}^{2}\right) .
$$

Une telle surestimation est évidemment inacceptable !

\subsection{Deuxième exemple}

Fondation de largeur

Surcharge nulle

$B=40 \mathrm{~m}$

Couche d'épaisseur

$q=0$

Cohésion en surface

$h=7 \mathrm{~m}$

Gradient vertical

descendant

de cohésion

$\mathrm{C}^{o}=10^{4} \mathrm{~Pa}\left(\simeq 1 \mathrm{t} / \mathrm{m}^{2}\right)$

$g^{o}=5 \times 10^{2} \mathrm{~N} / \mathrm{m}^{3}$

$\left(\simeq 0.05 \mathrm{t} / \mathrm{m}^{3}\right)$

D'où

$$
g^{\circ} \mathrm{B} / \mathrm{C}^{o}=2 \text { et } \mathrm{B} / h=5.7
$$

On se reporte à l'abaque de la figure 18. Dans ce cas, on a $\mathrm{B} / h>(\mathrm{B} / h)_{0}=2.8$, et on lit :

$$
\begin{aligned}
& \mu_{c}=1.1 \\
& \mathrm{~N}_{c}^{\prime}=7 \quad\left(\mathrm{~N}_{c}^{\prime}=7 \operatorname{par}(2.2)\right)
\end{aligned}
$$

d'où :

$$
p_{\text {ult }}=1.1 \times 10^{4}(7+0.5) \mathrm{Pa}
$$

soit :

$$
p_{\text {tat }}=8.25 \times 10^{4} \mathrm{~Pa}\left(=8.41 \mathrm{t} / \mathrm{m}^{2}\right) .
$$

Par la formule (7.2) on obtiendrait :

$$
\bar{p}_{\text {ult }}=7 \times 10^{4}(1+1 / 5.7) \mathrm{Pa}
$$

d'où :

$$
\bar{p}_{\text {ult }}=8.23 \times 10^{4} \mathrm{~Pa}\left(=8.39 \mathrm{t} / \mathrm{m}^{2}\right) .
$$

On voit que cette fois la concordance entre les résultats fournis par les deux calculs est excellente, mais on remarque également que l'utilisation de la formule exacte ne présente pas plus de difficultés, voire même moins, que l'utilisation de la formule approchée (7.2). 


\section{BIBLIOGRAPHIE}

[1] BERTHET (D.), HAYOT (J.-C.) et SALENÇON (J.) (1972). - Poinçonnement d'un milieu semiinfini en matériau plastique de Tresca non homogène. Archives of Mechanics, vol. 24, $\mathrm{n}^{\circ}$ 1, pp. 127-138.

[2] BISHOP (J.F.W.) (1953), - On the complete solution to problems of deformation of a plastic rigid material. Jnl Mech. Phys. Solids, vol. 2, $\mathrm{n}^{\circ} 1$, pp. 43-53.

[3] BUTTON (S.J.) (1953). - The bearing capacity of footings on a two-layer cohesive subsoil. Proc. 3rd Int. Conf. Soil Mech., Zurich, vol. 1 , pp. 332-335.

[4] CROC (M.), MICHEL (G.) et PECKER (A.) (1972). - Quelques problèmes de non-homogénéité en symétrie axiale. Travail de fin d'études E.N.P.C. Paris, Laboratoire de Mécanique des Solides, Paris.

[5] DAVIS (E.H.) et BOOKER (J.R.) (1973). The effect of increasing strength with depth on the bearing capacity of clays. Géotechnique, vol. $23, \mathrm{n}^{\circ} 4$, pp. 551-563.

[6] EASON (G.) et SHIELD (R.T.) (1960), - The plastic indentation of a semi-infinite solid by a perfectly rough circular punch. Z.A.M.P., vol. $11, \mathrm{n}^{\circ} 1$, pp. 33-43.

[7] FAVRETTl (G.) (1965). - Impronta di un punzone rigido su un materiale non omogeneo. Ingegn. Mecc., vol 14, no 9, pp. 37-50.

[8] FAVRETTI (G.) (1965). - Dipendenza fra durezza e profondità di cimentazione. Applicazione della teoria della plasticità dello studio del problema. Ingegn. Mecc., vol 15, $\mathrm{n}^{\circ} 6$.

[9] FAVRETTI (G.) (1966). - Indentation of a rigid punch on a platically non homogeneous material. Meccanica, vol. 1, n ${ }^{\circ}$ 3/4, pp. 83-94.

[10] GIROUD (J.-P.), TRAN-VÔ-NHIEM et OBIN (J.-P.) (1973). - Tests pour le calcul des fondations. Tome III, Dunod, Paris.

[11] JOHNSON (W.) et KUDO (H.) (1960). - The compression of a rigid perfectly plastic material between rough parallel dies of unequal width. Int. Inl. Mech. Sc., vol. 1, no 4, p. 336.
[12] KUZNETZOV (A.I.) ((1958). - Déformation plane des corps plastiques non homogènes (en russe) Vestnik Leningrad Univ. Ser. Mat. Mekh Astr, vol 3, pp. 112-131.

[13] KUZNETZOV (A.I.) (1958). - The problem of torsion and plane strain of non-homogeneous body. Arch. Mech. Stos. vol. 10, no 4, pp. 447 . 462.

[14] MEYERHOF (G.G.) et CHAPLIN (T.K.) (1953). - The compression and bearing capacity of cohesive layers. $\mathrm{Br}$. Jnl, Appl. Phys., vol. 4, 20.

[15] MANDEL (J.) et SALENÇON (J.) (1972). Force portante d'un sol sur une assise rigide (étude théorique). Géotechnique, vol. 22, n ${ }^{\circ} 1$, pp. 79-93.

[16] MATAR (M.) (1976). - Capacité portante d'une semelle filante sur une couche de sol d'épaisseur limitée et de cohésion variable avec la profondeur. Travail de fin d'études E.N.P.C., Paris, Laboratoire de Mécanique des Solides, Palaiseau.

[17] OLSZAK (W.), RYCHLEWSKI (J.) et URBANOWSKI (W.) (1962). - Plasticity under nonhomogeneous conditions. Advances in Applied Mechanics. Ac. Press. N.Y., pp. 132-214.

[18] OSTROWSKA (J.) (1968). - Initial plastic flow of a semi-space with a strong layer non homogeneity. Arch. Mech. Stos., vol. 20, no 6, pp. 651-668.

[19] SALENÇON (J.) (1968). - Etude d'une classe de solutions cinématiques pour le problème du poinçonnement d'un demi-plan non homogène. C.R. Ac. Sc., Paris, série A, t. 267, pp. 171-173.

[20] SALENÇON (J.) (1969). - La théorie des charges limites dans la résolution des problèmes de plasticité en déformation plane. Thèse Dr. es Sc. Univ., Paris.

[21] SALENÇON (J.), CROC (M.), MICHEL (G.) et PECKER (A.) (1973), - Force portante d'une fondation de révolution sur un bicouche. C.R. Ac. Sc. Paris, série A, t. 276, pp. 1569-1572.

[22] SALENÇON (J.) (1974). - Bearing capacyti of a footing on a $\Phi=0$ soil with linearly varying shear strength. Géotechnique, vol. 24, $\mathrm{n}^{\circ} 3$, pp. $443-446$. 
[23] SALENÇON (J.) (1974). - Théorie de la plasticité pour les applications à la mécanique des sols. Eyrolles, Paris.

[24] SALENÇON (J.) (1976). - Calcul à la rupture et plasticité. Cours E.N.P.C., Paris.

[25] SALENÇON (J.), FLORENTIN (P.) et GABRIEL (Y.) (1976). - Capacité portante globale d'une fondation sur un sol non-homogène. Géotechnique, vol. 26, n 2, pp. 351-370.
[26] SAYIR (M.) et ZIEGLER (H.) (1968), - Zum Prandtlschhen Stampelproblem. Ingenieur Archiv. vol. 36, n ${ }^{\circ}$ 5, pp. 294-302.

[27] SPENCER (A.J.M.) (1961). - Perturbation method in Plasticity. I. Plane strain of non homogeneous plastic solids : J. Mech. Phys. Solids, vol. $9, \mathrm{n}^{\circ} 4$, pp. $279-288$.

[28] SHIELD (R.T.) (1954). - Plastic potential and Prandtl bearing capacity solution. Il Appl. Mech. trans. ASME, vol. 21, pp. 193-194. 